\title{
Some Invariants of Circulant Graphs
}

\author{
Mobeen Munir ${ }^{1}$, Waqas Nazeer ${ }^{1}$, Zakia Shahzadi ${ }^{1}$ and Shin Min Kang ${ }^{2,3, *}$ \\ 1 Division of Science and Technology, University of Education, Lahore 54000, Pakistan; \\ mmunir@ue.edu.pk (M.M.); nazeer.waqas@ue.edu.pk (W.N.); zakiashahzadi@yahoo.com (Z.S.) \\ 2 Department of Mathematics and Research Institute of Natural Science, Gyeongsang National University, \\ Jinju 52828, Korea \\ 3 Center for General Education, China Medical University, Taichung 40402, Taiwan \\ * Correspondence: smkang@gnu.ac.kr; Tel.: +82-55-772-1420
}

Academic Editor: Angel Garrido

Received: 9 October 2016; Accepted: 11 November 2016; Published: 18 November 2016

\begin{abstract}
Topological indices and polynomials are predicting properties like boiling points, fracture toughness, heat of formation, etc., of different materials, and thus save us from extra experimental burden. In this article we compute many topological indices for the family of circulant graphs. At first, we give a general closed form of M-polynomial of this family and recover many degree-based topological indices out of it. We also compute Zagreb indices and Zagreb polynomials of this family. Our results extend many existing results.
\end{abstract}

Keywords: circulant graphs; topological indices; polynomials

\section{Introduction}

A number, polynomial or a matrix can uniquely identify a graph. A topological index is a numeric number associated to a graph which completely describes the topology of the graph, and this quantity is invariant under the isomorphism of graphs. The degree-based topological indices are derived from degrees of vertices in the graph. These indices have many correlations to chemical properties. In other words, a topological index remains invariant under graph isomorphism.

The study of topological indices, based on distance in a graph, was effectively employed in 1947 in chemistry by Weiner [1]. He introduced a distance-based topological index called the "Wiener index" to correlate properties of alkenes and the structures of their molecular graphs. Recent progress in nano-technology is attracting attention to the topological indices of molecular graphs, such as nanotubes, nanocones, and fullerenes to cut short experimental labor. Since their introduction, more than 140 topological indices have been developed, and experiments reveal that these indices, in combination, determine the material properties such as melting point, boiling point, heat of formation, toxicity, toughness, and stability [2]. These indices play a vital role in computational and theoretical aspects of chemistry in predicting material properties [3-8].

Several algebraic polynomials have useful applications in chemistry, such as the Hosoya Polynomial (also called the Wiener polynomial) [9]. It plays a vital role in determining distance-based topological indices. Among other algebraic polynomials, the M-polynomial—introduced recently in 2015 [10]—plays the same role in determining the closed form of many degree-based topological indices. Other famous polynomials are the first Zagreb polynomial and the second Zagreb polynomial.

A graph $G$ is an ordered pair $(V, E)$, where $V$ is the set of vertices and $E$ is the set of edges. A path from a vertex $v$ to a vertex $w$ is a sequence of vertices and edges that starts from $v$ and stops at $w$. The number of edges in a path is called the length of that path. A graph is said to be connected if there is a path between any two of its vertices. The distance $d(u, v)$ between two vertices $u, v$ of a connected graph $G$ is the length of a shortest path between them. Graph theory is contributing a lion's share in 
many areas such as chemistry, physics, pharmacy, as well as in industry [11]. We will start with some preliminary facts.

Let $G$ be a simple connected graph and let $u v$ represent the edge between the vertices $u$ and $v$. The number of vertices of $G$, adjacent to a given vertex $v$, is the "degree" of this vertex, and will be denoted by $d_{v}$. We define $V_{k}=\left\{v \in V(G) \mid d_{v}=k\right\}, E_{i, j}=\left\{u v \in E(G) \mid d_{u}=j\right.$ and $\left.d_{v}=i\right\}$, $\delta=\operatorname{Min}\left\{d_{v} \mid v \in V(G)\right\}, \triangle=\operatorname{Max}\left\{d_{v} \mid v \in V(G)\right\}$, and $m_{i j}$ as the number of edges $u v$ of $G$ such that $\left\{d_{v}, d_{u}\right\}=\{i, j\}$. The M-polynomial of $G$ is defined as:

$$
M(G, x, y)=\sum_{\delta \leq i \leq j \leq \triangle} m_{i j} x^{i} y^{j}
$$

Active research is in progress, and many authors computed M-polynomials for different types of nonmaterial, for example see [12-16] and the references therein.

The Wiener index of $G$ is defined as:

$$
W(G)=\frac{1}{2} \sum_{(u, v)} d(u, v)
$$

where $(u, v)$ is any ordered pair of vertices in G. Gutman and Trinajstić [11] introduces important topological index called first Zagreb index, denoted by $M_{1}(G)$, and is defined as:

$$
M_{1}(G)=\sum_{u v \in E(G)}\left(d_{u}+d_{v}\right)
$$

The second Zagreb index $M_{2}(G)$ and the second modified Zagreb index ${ }^{m} M_{2}(G)$ are defined as:

$$
M_{2}(G)=\sum_{u v \in E(G)}\left(d_{u} \times d_{v}\right)
$$

and:

$$
{ }^{m} M_{2}(G)=\sum_{u v \in E(G)} \frac{1}{d_{u} \cdot d_{v}}
$$

Results obtained in the theory of Zagreb indices are summarized in the review [17].

In 1998, working independently, Bollobas and Erdos [18] and Amic et al. [19] proposed general Randić index. It has been extensively studied by both mathematicians and theoretical chemists (see, for example, [20,21]). The Randić index denoted by $R_{\alpha}(G)$ is the sum of $\left(d u d_{v}\right)^{\alpha}$; i.e.:

$$
R_{\alpha}(G)=\sum_{u v \in E(G)}\left(d u d_{v}\right)^{\alpha}
$$

where $\alpha$ is any constant.

The symmetric division index is defined by:

$$
\operatorname{SDD}(G)=\sum_{u v \in E(G)}\left(\frac{\min \left\{d_{u}, d_{v}\right\}}{\max \left\{d_{u}, d_{v}\right\}}+\frac{\max \left\{d_{u}, d_{v}\right\}}{\min \left\{d_{u}, d_{v}\right\}}\right)
$$

These indices can help to characterize the chemical and physical properties of molecules (see [9]).

Table 1 enlists some standard degree-based topological indices and their derivation from M-polynomial [10]. 
Table 1. Derivation of topological indices from M-polynomial.

\begin{tabular}{ccc}
\hline Topological Index & $f(x, y)$ & Derivation from $\boldsymbol{M}(\boldsymbol{G}, \boldsymbol{x}, \boldsymbol{y})$ \\
\hline First Zagreb & $x+y$ & $\left.\left(D_{x}+D_{y}\right)(M(G ; x, y))\right|_{x=y=1}$ \\
Second Zagreb & $x y$ & $\left.\left(D_{x} D_{y}\right)(M(G ; x, y))\right|_{x=y=1}$ \\
${ }^{m} M_{2}(G)$ & $\frac{1}{x y}$ & $\left.\left(S_{x} D_{y}\right)(M(G ; x, y))\right|_{x=y=1}$ \\
General Randić $\alpha \in N$ & $(x y)^{\alpha}$ & $\left.\left(D_{x}^{\alpha} D_{y}^{\alpha}\right)(M(G ; x, y))\right|_{x=y=1}$ \\
General Randić $\alpha \in N$ & $\frac{1}{x y}$ & $\left.\left(S_{x}^{\alpha} S_{y}^{\alpha}\right)(M(G ; x, y))\right|_{x=y=1}$ \\
Symmetric Division Index & $\frac{x^{2}+y^{2}}{x y}$ & $\left.\left(D_{x} S_{y}+D_{y} S_{x}\right)(M(G ; x, y))\right|_{x=y=1}$ \\
\hline
\end{tabular}

Where $D_{x}(f(x, y))=x \frac{\partial f(x, y)}{\partial x}, D_{y}(f(x, y))=y \frac{\partial f(x, y)}{\partial y}, S_{x}(f(x, y))=\int_{0}^{x} \frac{f(t, y)}{t} d t, S_{y}(f(x, y))=\int_{0}^{y} \frac{f(x, t)}{t} d t$. For a simple connected graph, the first Zagreb polynomial is defined as:

$$
M_{1}(G, x)=\sum_{u c \in E(G)} x^{\left[d_{u}+d_{v}\right]}
$$

and the second Zagreb polynomial is defined as:

$$
M_{2}(G, x)=\sum_{u c \in E(G)} x^{\left[d_{u} \times d_{v}\right]}
$$

In 2013, Shirdel et al. in [22] proposed the hyper-Zagreb index, which is also degree-based, given as:

$$
H M(G)=\sum_{u c \in E(G)}\left[d_{u}+d_{v}\right]^{2}
$$

In 2012, Ghorbani and Azimi [23] proposed two new variants of Zagreb indices; namely, the first multiple Zagreb index $P M_{1}(G)$ and the second multiple Zagreb index $P M_{2}(G)$, which are defined as:

$$
\begin{aligned}
& P_{1}(G)=\prod_{u v \in E(G)}\left[d_{u}+d_{v}\right] \\
& P M_{2}(G)=\prod_{u v \in E(G)}\left[d_{u} \times d_{v}\right]
\end{aligned}
$$

In this paper, we address the family of circulant graphs. We give closed forms of M-polynomial and Zagreb Polynomials for this family. We also compute many degree-based topological indices.

Definition 1. Let $n, m$, and $a_{1}, \ldots, a_{m}$ be positive integers, where $1 \leq a_{i} \leq\left\lfloor\frac{n}{2}\right\rfloor$ and $a_{i} \neq a_{j}$ for all $1 \leq i<j \leq m$. An undirected graph with the set of vertices $V=\left\{v_{1}, \ldots, v_{n}\right\}$ and the set of edges $E=\left\{v_{i} v_{i+a_{j}}: 1 \leq i \leq n, 1 \leq j \leq m\right\}$, where the indices being taken modulo $n$, is called the circulant graph, and is denoted by $C_{n}\left(a_{1}, \ldots, a_{m}\right)$.

The graph of $C_{11}(1,2,3)$ is shown in Figure 1 .

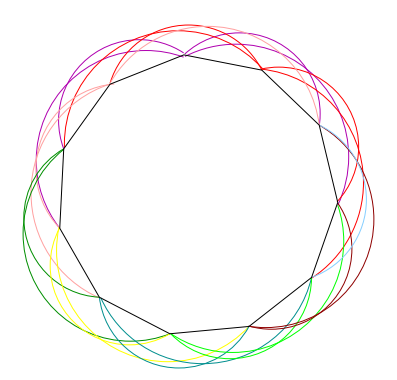

Figure 1. $C_{11}(1,2,3)$. 
This is one of the most comprehensive families, as its specializations give some important families. Classes of graphs that are circulant include the Andrásfai graphs, antiprism graphs, cocktail party graphs, complete graphs, complete bipartite graphs, crown graphs, empty graphs, rook graphs, Möbius ladders, Paley graphs of prime order, prism graphs, and torus grid graphs. Special cases are summarized in the Table 2.

Table 2. Special cases of circulant graphs.

\begin{tabular}{cccc}
\hline Graph & Symbol & Graph & Symbol \\
\hline 2-path graph & $C_{i_{2}}(1)$ & triangle graph & $C_{i_{3}}(1)$ \\
square graph & $C_{i_{4}}(1)$ & tetrahedral graph & $C_{i_{4}}(1,2)$ \\
5-cycle graph & $C_{i_{5}}(1)$ & pentatope graph & $C_{i_{5}}(1,2)$ \\
6-cycle graph & $C_{i_{6}}(1)$ & octahedral graph & $C_{i_{6}}(1,2)$ \\
utility graph & $C_{i_{6}}(1,3)$ & 3-prism graph & $C_{i_{6}}(2,3)$ \\
6-complete graph & $C_{i_{6}}(1,2,3)$ & 7-cycle graph & $C_{i_{7}}(1)$ \\
7-complete graph & $C_{i_{7}}(1,2,3)$ & 8-cycle graph & $C_{i_{8}}(1)$ \\
4-antiprism graph & $C_{i_{8}}(1,2)$ & (4,4)-complete bipartite graph & $C_{i_{8}}(1,3)$ \\
4-Möbius ladder graph & $C_{i_{8}}(1,4)$ & 16-cell graph & $C_{i_{8}}(1,2,3)$ \\
8-complete graph & $C_{i_{8}}(1,2,3,4)$ & 9-cycle graph & $C_{i_{9}}(1)$ \\
9-complete graph & $C_{i_{9}}(1,2,3,4)$ & 10-cycle graph & $C_{i_{10}}(1)$ \\
5-antiprism graph & $C_{i_{10}}(1,2)$ & 5-crown graph & $C_{i_{10}}(1,3)$ \\
5-Möbius ladder graph & $C_{i_{10}}(1,5)$ & 5-prism graph & $C_{i_{10}}(2,5)$ \\
5-cocktail party graph & $C_{i_{10}}(1,2,3,4)$ & (5,5)-complete bipartite graph & $C_{i_{10}}(1,3,5)$ \\
\hline
\end{tabular}

Because of this somewhat universality, circulant graphs have been the subject of much investigation; for example, the chromatic index for circulant graphs is computed in [24]. Connectivity is discussed in [25], and the Weiner index is computed in [26]. Exact values of the domination number of some families of circulant graphs are given in [27]. Habibi et. al. computed the revised Szeged spectrum of circulant graphs [28]. Multi-level and antipodal labelings for circulant graphs is discussed in $[29,30]$.

\section{Main Theorem}

We divided our main results into two parts.

\subsection{Polynomials}

In this section, we computed the closed forms of some polynomials.

Theorem 1. Let $C_{n}\left(a_{1}, a_{2}, \ldots, a_{m}\right)$ be a circulant graph. Then, the M-Polynomial is:

$$
M\left(\left(C_{n}\left(a_{1}, a_{2}, \ldots, a_{m}\right), x, y\right)=n x^{n-1} y^{n-1}\right.
$$

Proof. Let $C_{n}\left(a_{1}, a_{2}, \ldots, a_{m}\right)$, where $n=3,4 \ldots . n$. and $1 \leq a_{i} \leq\left\lfloor\frac{n}{2}\right\rfloor$ and $a_{i} \neq a_{j}$ when $n=$ even and when $1 \leq a_{i} \leq\left\lfloor\frac{n}{2}\right\rfloor$ and $a_{i}<a_{j}$ when $n=$ odd be the circulant graph. From the structure of $C_{n}\left(a_{1}, a_{2}, \ldots, a_{m}\right)$, we can see that there is one partition $V_{\{1\}}=\left\{v \in V\left(C_{n}\left(a_{1}, a_{2}, \ldots, a_{m}\right)\right) \mid d_{v}=n\right\}$. We see that the edge set of $C_{n}\left(a_{1}, a_{2}, \ldots, a_{m}\right)$ partitions as follows:

$$
E_{\{n-1, n-1\}}=\left\{e=u v \epsilon E\left(C_{n}\left(a_{1}, a_{2}, \ldots, a_{m}\right)\right) \mid d_{u}=n-1 \& d_{v}=n-1\right\} \rightarrow\left|E_{\{n-1, n-1\}}\right|=n
$$


Thus, the M-Polynomial of $\left(C_{n}\left(a_{1}, a_{2}, \ldots, a_{m}\right), x, y\right)$ is:

$$
\begin{aligned}
M\left(C_{n}\left(a_{1}, a_{2}, \ldots, a_{m}\right), x, y\right) & =\sum_{i \leq j} m_{i j}\left(C_{n}\left(a_{1}, a_{2}, \ldots, a_{m}\right)\right) x^{i} y^{j} \\
& =\sum_{n-1 \leq n-1} m_{n-1 \times n-1}\left(C_{n}\left(a_{1}, a_{2}, \ldots, a_{m}\right)\right) x^{n-1} y^{n-1} \\
& =\sum_{u v \in E_{\{n-1, n-1\}}} m_{n-1 \times n-1}\left(C_{n}\left(a_{1}, a_{2}, \ldots, a_{m}\right)\right) x^{n-1} y^{n-1} \\
& =\left|E_{\{n-1, n-1\}}\right| x^{n-1} y^{n-1} \\
& =n x^{n-1} y^{n-1}
\end{aligned}
$$

In the following theorem, we computed first and second Zagreb polynomials.

Theorem 2. Let $C_{n}\left(a_{1}, a_{2}, \ldots, a_{m}\right)$ be a circulant graph. Then:

(1) $M_{1}\left(C_{n}\left(a_{1}, a_{2}, \ldots, a_{m}\right), x\right)=n x^{2(n-1)}$

(2) $\quad M_{2}\left(C_{n}\left(a_{1}, a_{2}, \ldots, a_{m}\right), x\right)=n x^{(n-1)^{2}}$

Proof. Let $C_{n}\left(a_{1}, a_{2}, \ldots, a_{m}\right)$ be a complete circulant graph. The edge set of $C_{n}\left(a_{1}, a_{2}, \ldots, a_{m}\right)$ has one partition based on degree of vertices. The edge partition has $n$ edges $u v$, where $d_{u}=d_{v}=n-1$. It is easy to see that $\mid E_{1}\left(C_{n}\left(a_{1}, a_{2}, \ldots, a_{m}\right) \mid=d_{n-1 \times n-1}\right.$. Now we have:

(1)

$$
\begin{aligned}
M_{1}\left(C_{n}\left(a_{1}, a_{2}, \ldots, a_{m}\right)\right. & =\sum_{u v \in E\left(C_{n}\left(a_{1}, a_{2}, \ldots, a_{m}\right)\right.} x^{\left[d_{u}+d_{v}\right]}, \\
& =\sum_{u v \in E_{1}\left(C_{n}\left(a_{1}, a_{2}, \ldots, a_{m}\right)\right.} x^{\left[d_{u}+d_{v}\right]} \\
& =\mid E_{1}\left(C_{n}\left(a_{1}, a_{2}, \ldots, a_{m}\right) \mid x^{2(n-1)}\right. \\
& =n x^{2(n-1)}
\end{aligned}
$$

(2)

$$
\begin{aligned}
M_{2}\left(C_{n}\left(a_{1}, a_{2}, \ldots, a_{m}\right)\right. & =\sum_{u v \in E\left(C_{n}\left(a_{1}, a_{2}, \ldots, a_{m}\right)\right.} x^{\left[d_{u} \times d_{v}\right]} \\
& =\sum_{u v \in E_{1}\left(C_{n}\left(a_{1}, a_{2}, \ldots, a_{m}\right)\right.} x^{\left[d_{u} \times d_{v}\right]} \\
& =\mid E_{1}\left(C_{n}\left(a_{1}, a_{2}, \ldots, a_{m}\right) \mid x^{(n-1)^{2}}\right. \\
& =n x^{(n-1)^{2}}
\end{aligned}
$$

\subsection{Topological Indices}

In this section, we will recover some topological indices from polynomials computed in the above section.

Theorem 3. For the circulant graph $C_{n}\left(a_{1}, a_{2}, \ldots, a_{m}\right)$, we have:

(1) $M_{1}\left(C_{n}\left(a_{1}, a_{2}, \ldots, a_{m}\right)\right)=2 n(n-1)$

(2) $M_{2}\left(C_{n}\left(a_{1}, a_{2}, \ldots, a_{m}\right)\right)=n(n-1)^{2}$

(3) ${ }^{m} M_{2}\left(C_{n}\left(a_{1}, a_{2}, \ldots, a_{m}\right)\right)=\frac{n}{(n-1)^{2}}$ 
(4) $R_{\alpha}\left(C_{n}\left(a_{1}, a_{2}, \ldots, a_{m}\right)\right)=n\left\{(n-1)^{2}\right\}^{\alpha}$

(5) $R_{\alpha}\left(C_{n}\left(a_{1}, a_{2}, \ldots, a_{m}\right)\right)=\frac{n}{(n-1)^{\alpha}}$

(6) $\operatorname{SDD}\left(C_{n}\left(a_{1}, a_{2}, \ldots, a_{m}\right)\right)=2 n$

Proof. Let $f(x, y)=M\left(\left(C_{n}\left(a_{1}, a_{2}, \ldots, a_{m}\right), x, y\right)=n x^{n-1} y^{n-1}\right.$. Then:

$$
\begin{gathered}
D_{x}(f(x, y))=n(n-1) x^{n-1} y^{n-1} \\
\left.\left(D_{x} f(x, y)\right)\left(M\left(C_{n}\left(a_{1}, a_{2}, \ldots, a_{m}\right) ; x, y\right)\right)\right|_{x=y=1}=n(n-1) \\
D_{y}(f(x, y))=n(n-1) x^{n-1} y^{n-1} \\
\left.\left(D_{x} f(x, y)\right)\left(M\left(C_{n}\left(a_{1}, a_{2}, \ldots, a_{m}\right) ; x, y\right)\right)\right|_{x=y=1}=n(n-1) \\
S_{x} f(x, y)=\frac{n}{n-1} x^{n-1} y^{n-1} \\
\left.\left(S_{x} f(x, y)\right)\left(M\left(C_{n}\left(a_{1}, a_{2}, \ldots, a_{m}\right) ; x, y\right)\right)\right|_{x=y=1}=\frac{n}{n-1} \\
S_{y} f(x, y)=\frac{n}{n-1} x^{n-1} y^{n-1} \\
\left.\left(S_{y} f(x, y)\right)\left(M\left(C_{n}\left(a_{1}, a_{2}, \ldots, a_{m}\right) ; x, y\right)\right)\right|_{x=y=1}=\frac{n}{n-1}
\end{gathered}
$$

(1) $M_{1}\left(C_{n}\left(a_{1}, a_{2}, \ldots, a_{m}\right)\right)$ :

$$
\left.\left(D_{x}+D_{y}\right) f(x, y)\right)\left.\left(M\left(C_{n}\left(a_{1}, a_{2}, \ldots, a_{m}\right) ; x, y\right)\right)\right|_{x=y=1}=2 n(n-1)
$$

(2) $M_{2}\left(C_{n}\left(a_{1}, a_{2}, \ldots, a_{m}\right)\right)$ :

$$
\left.\left(D_{x} D_{y}\right) f(x, y)\right)\left.\left(M\left(C_{n}\left(a_{1}, a_{2}, \ldots, a_{m}\right) ; x, y\right)\right)\right|_{x=y=1}=n(n-1)^{2}
$$

(3) ${ }^{m} M_{2}\left(C_{n}\left(a_{1}, a_{2}, \ldots, a_{m}\right)\right)$ :

$$
\left.\left(S_{x} S_{y}\right) f(x, y)\right)\left.\left(M\left(C_{n}\left(a_{1}, a_{2}, \ldots, a_{m}\right) ; x, y\right)\right)\right|_{x=y=1}=\frac{n}{(n-1)^{2}}
$$

(4) $R_{\alpha}\left(C_{n}\left(a_{1}, a_{2}, \ldots, a_{m}\right)\right)$ :

$$
\left.\left(D_{x}^{\alpha} D_{y}^{\alpha}\right) f(x, y)\right)\left.\left(M\left(C_{n}\left(a_{1}, a_{2}, \ldots, a_{m}\right) ; x, y\right)\right)\right|_{x=y=1}=n(n-1)^{2 \alpha}
$$

(5) $R_{\alpha}\left(C_{n}\left(a_{1}, a_{2}, \ldots, a_{m}\right)\right)$ :

$$
\left.\left(S_{x}^{\alpha} S_{y}^{\alpha}\right) f(x, y)\right)\left.\left(M\left(C_{n}\left(a_{1}, a_{2}, \ldots, a_{m}\right) ; x, y\right)\right)\right|_{x=y=1}=\frac{n}{(n-1)^{\alpha}}
$$

(6) $\operatorname{SDD}\left(C_{n}\left(a_{1}, a_{2}, \ldots, a_{m}\right)\right)$ :

$$
\left.\left(D_{x} S_{y}+D_{y} S_{x}\right)\left(M\left(C_{n}\left(a_{1}, a_{2}, \ldots, a_{m}\right) ; x, y\right)\right)\right|_{x=y=1}=2 n
$$

Theorem 4. Let $\left(C_{n}\left(a_{1}, a_{2}, \ldots, a_{m}\right)\right.$ be a circulant graph. Then:

(1) $P M_{1}\left(M_{n}\right)=2(n-1)^{n}$

(2) $P M_{2}\left(M_{n}\right)=\left\{(n-1)^{2}\right\}^{n}$

(3) $H M\left(M_{n}\right)=\{2(n-1)\}^{2}(n)$ 
Proof. Let $C_{n}\left(a_{1}, a_{2}, \ldots, a_{m}\right)$ be a complete circulant graph. The edge set of $C_{n}\left(a_{1}, a_{2}, \ldots, a_{m}\right)$ has one partition based on degree of vertices.The edge partition has $n$ edges $u v$, where $d_{u}=d_{v}=n-1$. It is easy to see that $\left|E_{1}\left(M_{n}\right)\right|=d_{(n-1) \times(n-1)}$. Now, we have:

(1)

$$
\begin{aligned}
\operatorname{PM}_{1}\left(C_{n}\left(a_{1}, a_{2}, \ldots, a_{m}\right)\right. & =\prod_{u v \in E\left(C_{n}\left(a_{1}, a_{2}, \ldots, a_{m}\right)\right.}\left[d_{u}+d_{v}\right] \\
& \left.=\prod_{u v \in E_{1}\left(C _ { n } \left(S_{i}\right.\right.}\right)\left[d_{u}+d_{v}\right] \\
& =\{2(n-1)\} \mid E_{1}\left(C_{n}\left(a_{1}, a_{2}, \ldots, a_{m}\right) \mid\right. \\
& =2(n-1)^{n}
\end{aligned}
$$

(2)

$$
\begin{aligned}
P M_{2}\left(C_{n}\left(a_{1}, a_{2}, \ldots, a_{m}\right)\right. & =\prod_{u v \in E\left(C _ { n } \left(S_{i}\right.\right.}\left[d_{u} \times d_{v}\right] \\
& =\prod_{u v \in E_{1}\left(C_{n}\left(a_{1}, a_{2}, \ldots, a_{m}\right)\right.}\left[d_{u} \times d_{v}\right] \\
& =\left\{(n-1)^{2}\right\}^{\mid E_{1}\left(C_{n}\left(a_{1}, a_{2}, \ldots, a_{m}\right) \mid\right.} \\
& =\left\{(n-1)^{2}\right\}^{n}
\end{aligned}
$$

(3)

$$
\begin{aligned}
H M\left(C_{n}\left(a_{1}, a_{2}, \ldots, a_{m}\right)\right. & =\sum_{u v \in E\left(C_{n}\left(a_{1}, a_{2}, \ldots, a_{m}\right)\right.}\left[d_{u}+d_{v}\right]^{2} \\
& =\sum_{u v \in E_{1}\left(C_{n}\left(a_{1}, a_{2}, \ldots, a_{m}\right)\right.}\left[d_{u}+d_{v}\right]^{2} \\
& =\{2(n-1)\}^{2} \mid E_{1}\left(C_{n}\left(a_{1}, a_{2}, \ldots, a_{m}\right) \mid\right. \\
& =\{2(n-1)\}^{2}(n)
\end{aligned}
$$

\section{Conclusions}

In this article, we computed many topological indices for the family of circulant graphs. At first we give a general closed form of M-polynomial of this family and recover many degree-based topological indices out of it. We also compute Zagreb indices and Zagreb polynomials of this family. Our results actually extend many existing results about crown graphs, Paley graphs, complete bipartite, Möbius Ladders, any many other families; see Table 2.

Acknowledgments: This research is supported by Gyeongsang National University, Jinju 52828, Korea. We are thankful to the reviewers for suggestions that really improve this paper.

Author Contributions: All authors contributed equally to the writing of this paper. All authors read and approved the final manuscript.

Conflicts of Interest: The authors declare no conflict of interest.

\section{References}

1. Wiener, H. Structural determination of paraffin boiling points. J. Am. Chem. Soc. 1947, 69, 17-20.

2. Katritzky, A.R.; Jain, R.; Lomaka, A.; Petrukhin, R.; Maran, U.; Karelson, M. Perspective on the Relationship between Melting Points and Chemical Structure. Cryst. Growth Design 2001, 1, 261-265.

3. Rucker, G.; Rucker, C. On topological indices, boiling points, and cycloalkanes. J. Chem. Inf. Comput. Sci. 1991, 39, 788 . 
4. Dobrynin, A.A.; Entringer, R.; Gutman, I. Wiener index of trees: Theory and applications. Acta Appl. Math. 2001, 66, 211-249.

5. Du, W.; Li, X.; Shi, Y. Algorithms and extremal problem on Wiener polarity index. MATCH Commun. Math. Comput. Chem. 2009, 62, 235-244.

6. Gutman, I.; Polansky, O.E. Mathematical Concepts in Organic Chemistry; Springer: New York, NY, USA, 1986.

7. Ma, J.; Shi, Y.; Yue, J. The wiener polarity index of graph products. Ars Combin. 2014, 116, 235-244.

8. Ma, J.; Shi, Y.; Wang, Z.; Yue, J. On wiener polarity index of bicyclic networks. Sci. Rep. 2016, 6, 19066.

9. Gutman, I. Some Properties of the Wiener Polynomial; Graph Theory Notes: New York, NY, USA, 1993; Volume 125, pp. 13-18.

10. Klavzar, S.; Deutsch, E. M-Polynomial and Degree-Based Topological Indices. Iran. J. Math. Chem. 2015, 6, 93-102.

11. Gutman, I.; Trinajstic, N. Graph theory and molecular orbitals total $\phi$-electron energy of alternant hydrocarbons. Chem. Phys. Lett. 1972, 17, 535-538.

12. Munir, M.; Nazeer, W.; Rafique, S.; Kang, S.M. M-polynomial and degree-based topological indices of Nano star dendrimers. Symmetry 2016, 8, 97.

13. Munir, M.; Nazeer, W.; Rafique, S.; Nizami, A.R.; Kang, S.M. M-polynomial and degree-based topological indices of Titania Nanotubes. Symmetry 2016, 8, 117.

14. Munir, M.; Nazeer, W.; Rafique, S.; Kang, S.M. M-polynomial and degree-based topological indices of Buckytubes. Symmetry 2016, submitted.

15. Kang, S.; Munir, M.; Nizami, A.; Shahzadi, Z.; Nazeer, W. Some Topological Invariants of the Möbius Ladder. Preprints 2016, 2016110040, doi:10.20944/preprints201611.0040.v1.

16. Munir, M.; Nazeer, W.; Rafique, S.; Nizami, A.; Kang, S.M. Some Computational Aspects of Triangular Boron Nanotubes. Symmetry 2016, doi:10.20944/preprints201611.0041.v1.

17. Gutman, I.; Das, K.C. The first Zagreb indices 30 years after. MATCH Commun. Math. Comput. Chem. 2004, $50,83-92$.

18. Bollobas, B.; Erdös, P. Graphs of extremal weights. Ars Combin. 1998, 50, 225-233.

19. Amic, D.; Beslo, D.; Lucic, B.; Nikolic, S.; Trinajstić, N. The Vertex-Connectivity Index Revisited. J. Chem. Inf Comput. Sci. 1998, 38, 819-822.

20. Kier, L.B.; Hall, L.H. Molecular Connectivity in Structure-Activity Analysis; John Wiley \& Sons: New York, NY, USA, 1986.

21. Li, X.; Gutman, I. Mathematical Aspects of Randic-Type Molecular Structure Descriptors; Mathematical Chemistry Monographs, No. 1; University of Kragujevac: Kragujevac, Serbia, 2006.

22. Shirdel, G.H.; Pour, H.R.; Sayadi, A.M. The hyper-Zagreb index of graph operations. Iran. J. Math. Chem. 2013, 4, 213-220

23. Ghorbani, M.; Azimi, N. Note on multiple Zagreb indices. Iran. J. Math. Chem. 2012, 3, 137-143.

24. Voigt, M.; Walther, H. On the chromatic number of special distance graphs. Discrete Math. 1991, 97, $395-397$.

25. Boesch, F.; Tindell, R. Circulants and their connectivity. J. Graph Theory 1984, 8, 487-499.

26. Zhou, H. The Wiener Index of Circulant Graphs. J. Chem. 2014, doi:10.1155/2014/742121.

27. Fu, X.L.; Yang, Y.S.; Jiang, B.Q. On the domination number of the circulant graphs $C(n ; 1,2), C(n ; 1,3)$ and $C(n ; 1,4)$. Ars Comb. 2011, 102, 173-182.

28. Habibi, N.; Ashrafi, A.R. On revised szeged spectrum of a graph. TAMKANG J. Math. 2014, 45, 375-387.

29. Kang, S.M.; Nazeer, S.; Kousar, I.; Nazeer, W.; Kwun, Y.C. Multi-level and antipodal labelings for certain classes of circulant graphs. J. Nonlinear Sci. Appl. 2016, 9, 2832-2845.

30. Nazeer, S.; Kousar, I.; Nazeer, W. Radio and radio antipodal labelings for circulant graphs $G(4 k+2 ; 1 ; 2)$. J. Appl. Math. Inf. 2015, 33, 173-183.

(C) 2016 by the authors; licensee MDPI, Basel, Switzerland. This article is an open access article distributed under the terms and conditions of the Creative Commons Attribution (CC-BY) license (http://creativecommons.org/licenses/by/4.0/). 